\title{
Equivalence Transformations among Ising Models
}

\author{
Yougang Feng \\ Guizhou University, Guiyang, China \\ Email: ygfeng45@aliyun.com
}

Received 5 November 2014; accepted 18 November 2014; published 14 January 2015

Copyright (C) 2015 by author and Scientific Research Publishing Inc.

This work is licensed under the Creative Commons Attribution International License (CC BY). http://creativecommons.org/licenses/by/4.0/

(c) (i) Open Access

\begin{abstract}
Using topology, fractal analysis and investigation of lattice formation process we find two types of equivalence transformations among Ising models: topological equivalence transformation and formation equivalence transformation. With the help of the transformations and the known data of the critical points of simple cubic (sc) lattice and planar square (sq) lattice we get directly the critical points for face-centered cubic (fcc) lattice, body-centered cubic (bcc) lattice and diamond (d) lattice. The transformation itself results no error in the calculation. Other than Monte Carlo method and series expansion approach the equivalence transformations help us simplify much more greatly the calculation of the critical points for the three-dimensional models and understand much more deeply the structural connection among Ising models.
\end{abstract}

\section{Keywords}

\section{Ising, Critical Point, Fractal, Equivalence Transformation}

\section{Introduction}

Fractal structure is a class of complex ordered structures in nature, which exhibits not simply a higher degree but an altogether different level of complexity. During the 1980s physicists tried to describe phenomena on fractal, they succeeded in calculating some of physical characteristics of fractals [1] [2]. A deterministic fractal is created by applying a rule of some sort interactively and indefinitely. A fractal is a self-similar geometric structure that looks alike on all length scales. The sub-block and the block in the Ising models are just such type of structures [3]. Our approach combining the fractal analysis and the solvable Gaussian model has been succeeded in calculating the critical points for two-and-three-dimensional Ising models and analyzing the fluctuation structure of the details at the critical temperature [3]-[5]. The explicit investigation of the information about these structures helps us think further that the macroscopic property of an Ising model at the critical temperature is an 
overall behavior of the collective motion of spins being in excitation state. According to solid state physics the excitation states consist of a series of elementary exciting units having definite energy quanta and relevant quasi-momenta, and have the quantum characteristics. We call these elementary excitations spin phonons [4] [5]. It is the behavior of spin phonons that determines thermodynamic properties of an Ising model at the critical temperature. In the reference [3] two three-dimensional models are facilitated by our theory. The research indicates that an exact solving of a lattice system depends strongly on the accurate analysis on its fractal structure. It is not necessary, then, to analyze the fractals for each lattice system if some equivalence relations among Ising models can be found that transfer some unknown fractal structure into those we have solved successfully.

Mentioning the equivalence transformation we may trace back to the 1940s, Kramers and Wannier discovered a transformation to enable them to get the critical point for square lattice [6]. Onsager pointed out their work to be topologic self-duality transformation [7]. He further discovered a star-triangle transformation, where a star consists of a central spin interacting three neighbor spins and can be transformed into a triangle of three spins interacting each other. The so called "decoration" or "iteration" transformation was discussed by Fisher [8], leading to solutions for further plane Ising nets and also for lattices in which the spins on alternate sites have a magnitude greater than $S=1 / 2$. However, all of three-dimensional lattice systems cannot be solved by his approach, although he found theoretically that there are some transformations between these systems such as a simple cubic lattice and a tetrahedron lattice, with which we are good familiar.

Solving of three-dimensional Ising models has more far-reaching significance because the actual ferromagnetic elements have different crystal textures; for example iron is body-centered cubic (bcc) while nickel is facecentered cubic (fcc). The investigation of their structures will help us deeply understand general laws of ferromagnetic. According to our investigation in the 3-dimensional models there is a unique irreducible lattice: the tetrahedron lattice [3]; others are reducible and should be divided into sub-blocks. There are two ways to research further: The first is to find out the fractal for the individual lattices one by one, except the sc we have solved, which requires us to divide correctly their sub-blocks; this may not be a normal way of affairs, especially for those composite lattices such as the $d$ lattice. Another way is to look for equivalence relations among the models, using which the fcc, bcc and d models can be described by the sc or the other such as the sq we have solved exactly.

In this paper we find two types of equivalence transformations: topological equivalence transformation and formation equivalence transformation, by means of which we get directly the critical points for the fcc, bcc and d lattice systems. In Section 2, we first introduce some new concepts then derive the two types of equivalence transformations. In Section 3, the two transformations are tested and verified and we further compare different theories of studying Ising models making use of the obtained data. The critical exponents are discussed simply. Section 4 is conclusion remark.

\section{Theory}

\section{1. $J$ and $J^{*}$}

In order to designate the relationship between the structure of a particular model and its critical temperature, and to compare the critical points for different models, it is convenient to unify their coupling constants, the applying of the normalized coupling constant is a wisdom choice. In terms of quantum mechanics the coupling constant is the exchange energy [9], it can be expressed in the form

$$
\iint u_{100}^{*}\left(\boldsymbol{r}_{1}\right) u_{200}^{*}\left(\boldsymbol{r}_{2}\right) \frac{e^{2}}{r_{12}^{2}} u_{100}\left(\boldsymbol{r}_{2}\right) u_{200}\left(\boldsymbol{r}_{1}\right) \mathrm{d}^{3} r_{1} \mathrm{~d}^{3} r_{2}
$$

The $u_{100}^{*}$ and $u_{200}^{*}$ are the conjugate states of the wavefunctions $u_{100}$ and $u_{200}$ which are the ground states of the first electron and the second electron, respectively, the $\boldsymbol{r}_{1}$ and $\boldsymbol{r}_{2}$ are the electrons position vectors, $e$ is the electron charge, $r_{12}$ the distance between the electrons. Clearly, the integral is independent of lattice structure. If the $r_{12}$ equals one unit length, Equation (1) is the expression of the normalized coupling constant $J$; while $r_{12}$ is smaller than one unit length, the integral is just the actual coupling constant $J^{*}$. From Equation (1), the relation between $J$ and $J^{*}$ is

$$
J=r_{12}^{2} J^{*}
$$




\section{2. $K_{c}$ and $K_{c}^{*}$}

A normalized critical point is defined as $K_{c}=J /\left(k_{B} T_{c}\right)$, where $k_{B}$ is Boltzmann constant, $T_{c}$ is the critical temperature. An equivalent critical point is defined as $K_{c}^{*}=J^{*} /\left(k_{B} T_{c}\right)$. Using Equation (2) we get

$$
K_{c}=r_{12}^{2} K_{c}^{*}
$$

For the fcc, $r_{12}=\sqrt{2} / 2$, so

$$
K_{c, f c c}=\frac{1}{2} K_{c, f c c}^{*}
$$

For the bcc, $r_{12}=\sqrt{3} / 2$, and

$$
K_{c, b c c}=\frac{3}{4} K_{c, b c c}^{*}
$$

For the sc, $r_{12}=1$, thus

$$
K_{c, s c}=K_{c, s c}^{*}
$$

It can be seen that different coupling constants provide different critical points for the same configuration, which critical temperature is unique. The normalized critical point for the sc lattice is just its equivalent critical point.

\subsection{Topological Equivalence}

The fcc lattice is a familiar structure for us, its structural diagram is often shown in the books on solid state physics. If we consider merely the nearest neighbor interaction the lattice can be considered a structure made up of infinite parallelepipeds, each of which is a primitive cell for the fcc. According to topology such structure is equivalence to the sc, we may call the fcc an equivalent sc. In a mathematics sense the topological equivalence model has the same fractals as the sc, which means that the sub-block and the block of the fcc are just the sc ones. Therefore, the equivalent critical point for the fcc can be represented by Equation (16) of the reference [3], thus

$$
K_{c, f c c}^{*}=K_{c, s c}
$$

In a similar way, the equivalent critical point for the bcc is given by

$$
K_{c, b c c}^{*}=K_{c, s c}
$$

Substitution of Equations (4) and (5) puts Equations (7) and (8) into

$$
K_{c, f c c}=\frac{1}{2} K_{c, s c}, K_{c, b c c}=\frac{3}{4} K_{c, s c}
$$

\subsection{Formation Equivalence}

In the sc system there are infinite horizontal planes parallel to one another and infinite vertical planes relatively parallel, each lattice belongs to not only one horizontal plane but also one vertical plane. Such structure means that the sc is a direct sum of the square lattices (sq) [10]. The definition of the fractal dimensions of the sq is given by the reference [3], if the fractal dimension of a sub-block of the sq is $D_{s q}$, the fractal dimension of a sub-block of the sc is $2 D_{s q}$ due to the direct sum relation, and the fractal dimension of the sc block is $2 D_{s q}^{2}$, where $D_{s q}^{2}$ is the dimension of the block of the sq. Therefore, the sc system consists of two independent subsystems, one has fractal dimension $2 D_{s q}$, another $2 D_{s q}^{2}$, and the critical point of the sc becomes [3]

$$
K_{c, s c}=\frac{1}{2\left(2 D_{s q}\right)}+\frac{1}{2\left(2 D_{s q}^{2}\right)}=\frac{1}{2} K_{c, s q}
$$

A composite lattice cannot be homeomorphism to a simple lattice such as the sc, so we should seek another way. The $\mathrm{d}$ lattice as a composite structure can be described as two interpenetrating fcc lattices displaced along 
the body diagonal of the conventional cube by one-fourth of the diagonal length. The nearest neighbor lattices make up a diamond primitive cell, which is a simple cubic with one lattice at the cube's center and the rest four lattices at its vertices, two of them on the top surface and the others on the bottom as shown by the figure 23 in the chapter 1 of the reference [11]. The nearest neighbor distance is $\sqrt{3} / 2$ of the cube side. Thus, we imagine that the formation equivalence for the $d$ lattice involves two steps. In the first step relating to a critical point $K_{c 1}$, two fcc lattices form a lattice structure, and they are independent of one another without interaction, so $K_{c 1}=2 K_{c, f c c}$. In the second step responsible for another critical point $K_{c 2}$ the nearest neighbor lattices form a primitive cell for the $d$ lattice. It is well known that the group IV elements like silicon and germanium crystallize in the $\mathrm{d}$ structures. An equivalent representation for the silicon structure is shown by the figure 19 and figure 20 in the chapter 8 of the reference [11]. The equivalent structure is viewed as a double planar square lattice, where one square lattice system lays overlap on another square lattice system, each lattice serves for the two systems at the same time. Considering the actual primitive cell for the $\mathrm{d}$ lattice is stereoscopic, we think therefore that the lattice should be a direct sum space of the two copies of the sq, being similar to the representation of Equation (10), except their nearest neighbor distance, which is $\sqrt{3} / 2$ of the body diagonal length of the cell. We then get $K_{c 2}=(1 / 2)(\sqrt{3} / 2)^{2} K_{c, s q}$. Therefore, the critical point of the d lattice spin system is written as

$$
K_{c, d}=K_{c 1}+K_{c 2}=2 K_{c, f c c}+\frac{3}{8} K_{c, s q}
$$

\section{Discussion}

There has been no way to solve exactly the three-dimensional Ising models so far, except our theory [3]. In order to examine the above equivalence transformations we should introduce those data concluded by other theories. The approach of the series expansion can give us the critical points with high accuracy. Lundow and his colleagues calculated the critical points for fcc, bcc and d lattices on the base of the computing the critical point $K_{c, s c}$ for the sc [12]: $K_{c, s c}=0.22165, K_{c, f c c}=0.102069, K_{c, b c c}=0.157371$, and $0.369678 \leq K_{c, d} \leq 0.369761$. Inserting the $K_{c, s c}=0.22165$ into Equations (9), (10), and (11), we have

$$
K_{c, f c c}=0.11083, K_{c, b c c}=0.16624, K_{c, d}=0.38789
$$

The magnitudes of the critical points in Equation (12) are greater than that they themselves compute, which may be related to the value of $K_{c, s c}$ they give. Sykes and his colleagues obtained another set of solutions for these lattices by means of the series expansion [13]: $K_{c, s c}=0.2217, K_{c, f c c}=0.1018$, and $K_{c, b c c}=0.1574$, without the datum $K_{c, d}$. Substitution $K_{c, s c}=0.2217$ gives us

$$
K_{c, f c c}=0.1109, K_{c, b c c}=0.1663
$$

The magnitudes of the data in Equation (13) are also lager than the author's. Using our theoretical results $K_{c, s c}=0.2150$ and $K_{c, s q}=0.4387$ [3], which we transfer into

$$
K_{c, f c c}=0.1075, K_{c, b c c}=0.1613, K_{c, d}=0.3795
$$

The behavior of the huge fluctuations attracted by a critical point shows the phase transition is irreversible, and the critical point is stable as being a minimum like a valley bottom between mountains. This critical property rules the principle of the method of series expansion in that the asymptotic value should finally go to a minimum after infinite iterating calculation. Such calculation, however, never been met in the practice, since the terms number in all of series expansions are always limited providing that the obtained values have to be regarded as results by man-made extension. This may be the cause that there are slight differences between the magnitudes of the critical points in Equation (14) from our theory and the ones out of the series expansions. An obvious example is the value of the $K_{c, d}$ given by Lundow, as an asymptotic minimum it should not be in between two values, about which the author pointed out that they could probably not aspire to the relatively high precision found in the case of bcc and fcc [12]. C. Domb get $K_{c, d}=0.369767786$ by the series expansion [14], which is close to ours given by Equation (14). The solutions $K_{c, d}=0.369685767$ and $K_{c, d}=0.36973980$ are found by the Monte Carlo technique and simultaneous analysis [15] [16], respectively, basically consist with Equation (14).

An equivalence transformation itself does not result in any error, which comes from the initial values of $K_{c, s c}$ and $K_{c, s q}$ through by Equations (7)-(9), and (11). Our theoretical values are $K_{c, s c}=0.2150$ and $K_{c, s q}=0.4387$ 
smaller than that other approaches or methods infer. By periodic boundary condition an infinite model can be broken up into parts, which we can recognize and solve exactly, and which fit together nicely in its original embedded space, namely, the model keeps intrinsically its topologic property. Under such condition the states in both positive infinity position and negative infinity position are uncertain. Inversely, the infinity boundary condition makes the states of a model in both the positions identity, this is an additional compulsory measure making the model never be embedded in its original space, namely, the topological structure of the model has changed [17]. This condition helped Wannier acquire $K_{c, s c}=0.4407$ greater than ours, in the meantime the planar square lattice had become into a torus square lattice [18]. The same boundary condition geometry problem can be found in Fisher's work [8].

The formation equivalence makes a composite lattice be simultaneously consistent of two or more lattice systems. Equations (9)-(11) lead to an algebraic expression $K_{c, d}=(7 / 4) K_{c, s c}$ for the d lattice, it is available for mathematics other than physics. Since it implies that the relevant nearest neighbor distance is $\sqrt{7} / 2$ greater than the lattice constant, such a nearest neighbor distance is impossible. The form $K_{c, d}=(7 / 8) K_{c, s q}$ by means of Equations (9)-(11) also has no physical meaning although it is applicable to the calculation of the critical point.

Finally, we discuss simply about the critical exponents, which are relative to the series expansions. They should be regarded as the variables describing phenomenologically the critical behaviors without referring to the critical fluctuation mechanism. As we have found out that the heat capacity of a three-dimensional Ising model at the critical temperature is attributed to four types of spin phonons originating in the sub-blocks, the ordered blocks, the lattices in the sub-blocks, and the lattices in the ordered blocks [4]. Such a complicated mechanism cannot be radically represented by a single exponent. In fact, the critical exponents appear initially in the early investigation of the critical phenomena, they do be the phenomenological variables.

\section{Conclusion Remark}

We find two types of equivalence transformations among Ising models: the topological equivalence transformation and the formation equivalence transformation. These transformations make us investigate effectively more Ising models in structures, especially for the three-dimensional ones. With the help of our approach we have obtained exact critical points for the bcc, fcc, and d lattice spin systems.

\section{References}

[1] Liu, S.H. (1986) Solid State Physics, 39, 207-273. http://dx.doi.org/10.1016/S0081-1947(08)60370-7

[2] Havlin, S. and Ben-Avraham, D. (1987) Advances in Physics, 36, 695-798. http://dx.doi.org/10.1080/00018738700101072

[3] Feng, Y.-G. (2014) American Journal of Modern Physics, 3, 184-194. http://dx.doi.org/10.11648/j.ajmp.20140304.16

[4] Feng, Y.-G. (2014) American Journal of Modern Physics, 3, 178-183. http://dx.doi.org/10.11648/j.ajmp.20140304.15

[5] Feng, Y.-G. (2014) American Journal of Modern Physics, 3, 211-217. http://dx.doi.org/10.11648/j.ajmp.20140306.11

[6] Kramers, H.A. and Wannier, G.H. (1941) Physical Review, 60, 252-262. http://dx.doi.org/10.1103/PhysRev.60.252

[7] Onsager, L. (1944) Physical Review, 65, 117-149. http://dx.doi.org/10.1103/PhysRev.65.117

[8] Fisher, M.E. (1968) Physical Review, 113, 969-981. http://dx.doi.org/10.1103/PhysRev.113.969

[9] Schiff, L.I. (1968) Quantum Mechanics. 3rd Edition, McGraw-Hill, New York.

[10] Fulton, W. (1995) Algebraic Topology. Springer-Verlag, New York. http://dx.doi.org/10.1007/978-1-4612-4180-5

[11] Kittel, C. (2005) Introduction to Solid State Physics. John Willey \& Sons, New York.

[12] Lundow, P.H., Markström, K. and Rosengren, A. (2009) Philosophical Magazine, 89, 2009-2042. http://dx.doi.org/10.1080/14786430802680512

[13] Sykes, M.F., Gaunt, D.S., Roberts, P.D. and Wyles, J.A. (1972) Journal of Physics A: General Physics, 5, 640-652. http://dx.doi.org/10.1088/0305-4470/5/5/005

[14] Domb, C. (1974) Ising Model. In: Domb, C. and Green, M.S., Eds., Phase Transitions and Critical Phenomena, Vol. 3, Academic Press, London, 357-484.

[15] Mouritsen, O.G. (1980) Journal of Physics C: Solid State Physics, 13, 3909. 
http://dx.doi.org/10.1088/0022-3719/13/20/013

[16] Deng, Y.J. and Blöte, H.W.J. (2003) Physical Review E, 68, Article ID: 036125. http://dx.doi.org/10.1103/PhysRevE.68.036125

[17] Feng, Y.G. (2005) Electronic Journal of Theoretical Physics, 7, 12-18.

[18] Wannier, G.H. (1945) Reviews of Modern Physics, 17, 50-60. http://dx.doi.org/10.1103/RevModPhys.17.50 
Scientific Research Publishing (SCIRP) is one of the largest Open Access journal publishers. It is currently publishing more than 200 open access, online, peer-reviewed journals covering a wide range of academic disciplines. SCIRP serves the worldwide academic communities and contributes to the progress and application of science with its publication.

Other selected journals from SCIRP are listed as below. Submit your manuscript to us via either submit@scirp.org or Online Submission Portal.
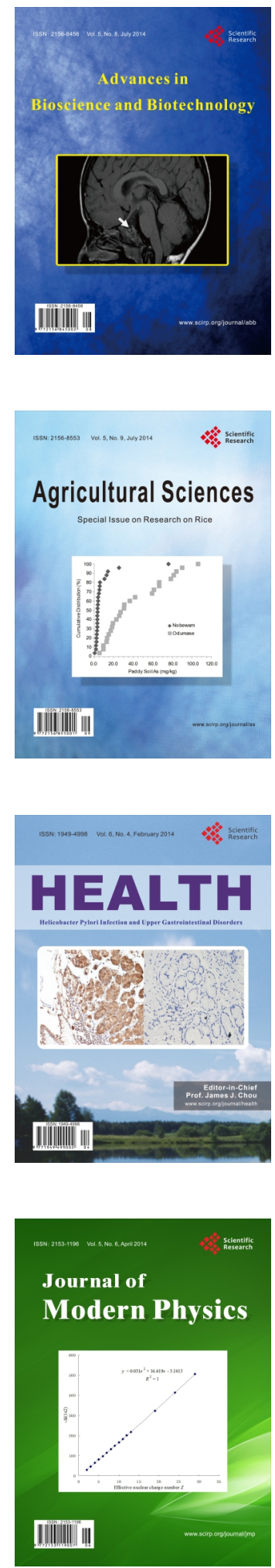
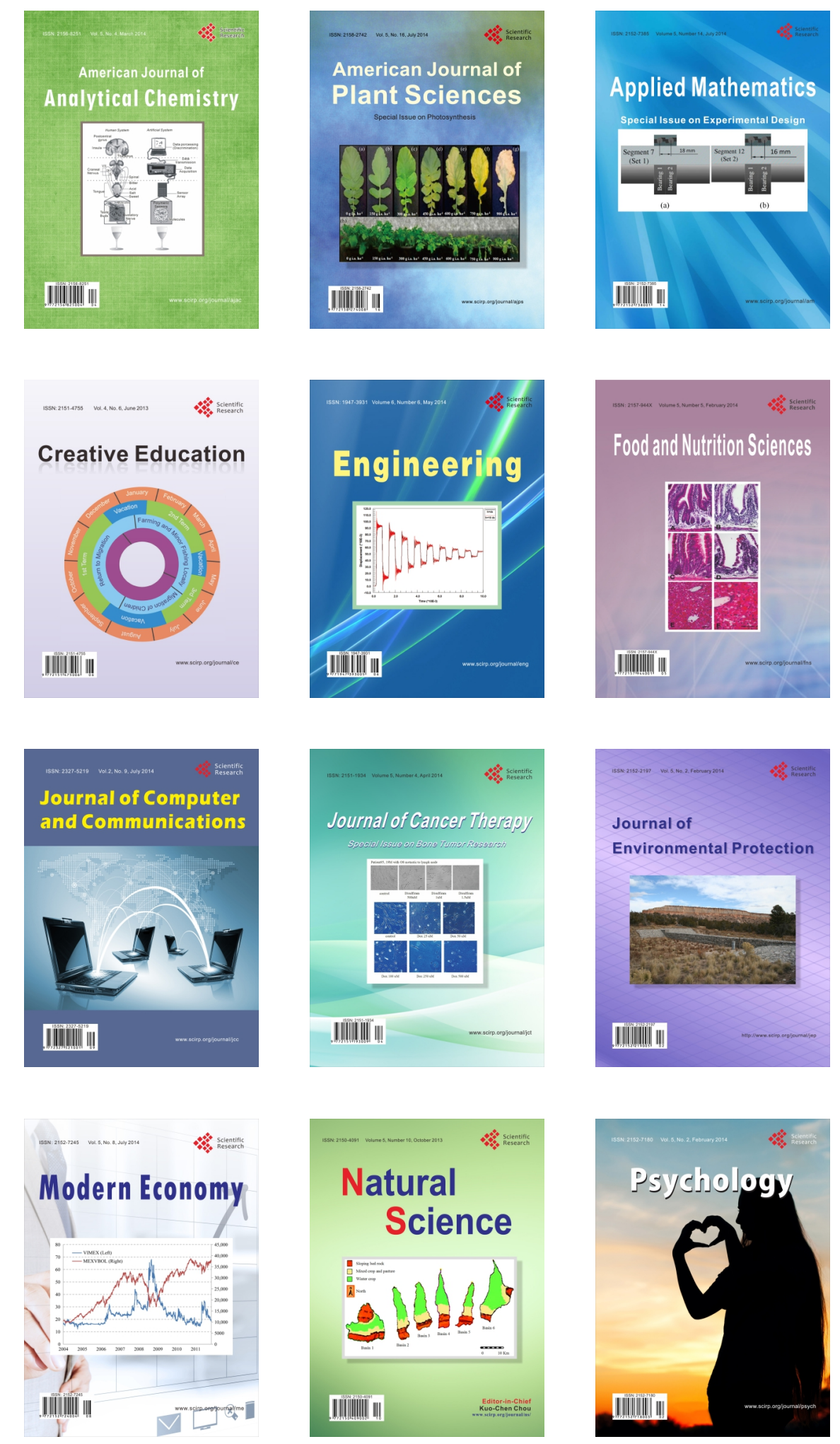